Casos Clínicos

Arch. Esp. Urol., 60, 7 (792-795), 2007

\section{ORQUITIS BRUCELAR. A PROPÓSITO DE UN NUEVO CASO Y REVISIÓN DE LA LITERATURA.}

José Antonio Cánovas Ivorra, Antonio Francisco Sánchez Díaz, Juan Salvador Fernández García, José Antonio García Zamora y Ángel Cuesta Climent.

Servicio de Urología. Hospital "Mare de Deu dels Lliris". Alcoy. Alicante. España.

Resumen.- OBJETIVO: Presentamos un caso de orquiepididimitis aguda unilateral secundaria a infección por Brucella y revisamos la literatura al respecto.

MÉTODOS: Presentamos el caso de un varón de 51 años con fiebre de predominio vespertino de un mes de evolución asociado a proceso inflamatorio subagudo en teste derecho y dolor osteoarticular costal izquierdo.

RESULTADOS: Se trata mediante Rifampicina a una dosis de $600 \mathrm{mg}$. / día durante 45 días y Doxiclina $100 \mathrm{mg}$. cada 12 horas durante 45 días.
CONCLUSIONES: Se debe tener en cuenta la etiología brucelósica ante orquiepididimitis de evolución tórpida, especialmente en áreas donde la Brucella pueda ser endémica y en personas que hayan tenido contacto con ganado o derivados de la leche no sujetos a control. Destacar que el contacto puede haber sido incluso varios meses antes de la clínica de orquiepididimitis.

Palabras clave: Orquiepididimitis. Brucella. Tratamiento.

Summary.- OBJECTIVE: We report one case of unilateral epididymo-orchitis secondary to Brucella and perform a bibliographic review.

METHODS: We report the case of a 51 -year-old male with fever, predominantly in the evening hours, over one month associated with a subacute inflammatory process on the right testicle and osteoarticular pain on the left ribs.

RESULTS: Treatment was performed with rifampicin 600 $\mathrm{mg} /$ day for 45 days and doxicilin $100 \mathrm{mg} / 12$ hours for 45 days.

CONCLUSIONS: The brucellar etiology should be taken into consideration in front of an epididymo- orchitis with torpid outcome, mainly in areas of endemic Brucella and patients that have had contact with cattle or non controlled milk derivatives. We emphasize that contact could have been several months before the appearance of clinical epididymoorchitis.

Keywords: Epididymo-orchitis. Brucella. Treatment.

\section{INTRODUCCIÓN}

La brucelosis es una zoonosis transmisible al ser humano y con capacidad de afectar a varios órganos y tejidos. La más frecuente en la especie humana es Brucella Mellitensis, cocobacilo Gram negativo, cuyo principal reservorio es el ganado. El principal mecanismo de contagio es vía oral y tras un periodo de incubación con alta variabilidad se suele presentar como síndrome febril y afectación de varios órganos y sistemas, entre los que figura el genitourinario con una frecuencia variable entre el 2 y el $20 \%$, con una típica manifestación, la orquiepididimitis unilateral o bilateral. Puede tener una evolución tórpida que cabe diferenciar de orquiepididimitis de otro origen como TBC. El tratamiento precoz puede evitar la orquiectomía y llegar a prevenir la afectación contralateral y las manifestaciones sistémicas. 


\section{CASO CLÍNICO}

Presentamos el caso de un paciente varón de 51 años de edad con antecedentes de Insuficiencia Venosa Periférica tratada mediante safenectomía. Trabajador del textil sin antecedentes de exposición sospechosos para la enfermedad. No más antecedentes de interés.

Acude al servicio de Urgencias por tumefacción escrotal subaguda derecha asociada a síndrome febril de 15 días de evolución tratada sin éxito por su médico de Atención Primaria con Quinolonas. La exploración es compatible con una orquiepididimitis aguda unilateral, y con tal antecedente decidimos la práctica de una ecografía escrotal que nos demuestra un área hipoecoica mal delimitada y de localización periférica (Figura 1). La analítica demuestra unos parámetros compatibles con la normalidad y el sedimento de orina fue normal. Se inicia tratamiento con Rifampicina a una dosis de 600 mg./día durante 45 días y Doxiclina 100 mg. cada 12 horas durante 45 días. La evolución fue satisfactoria.

Cuatro meses después, ingresa en el servicio de Reumatología tras ser visto en el Servicio de Urgencias, donde acude por un nódulo de consistencia blanda y características inflamatorias de varios días de evolución, con sensación distérmica, sin fiebre termometrada. A la exploración se aprecia una formación nodular dolorosa de localización atípica en parrilla costal izquierda. La analítica no mostraba leucositosis, ni anemia, tan solo un leve incremento de la P.C.R. El T.A.C. demuestra una asimetría de $3 \mathrm{~cm}$. de diámetro a nivel de la superficie costal 7 y $8^{\underline{a}}$.

La punción del nódulo y su posterior cultivo dan positivo para Brucella Mellitensis biotipo C, con Test de Rosa

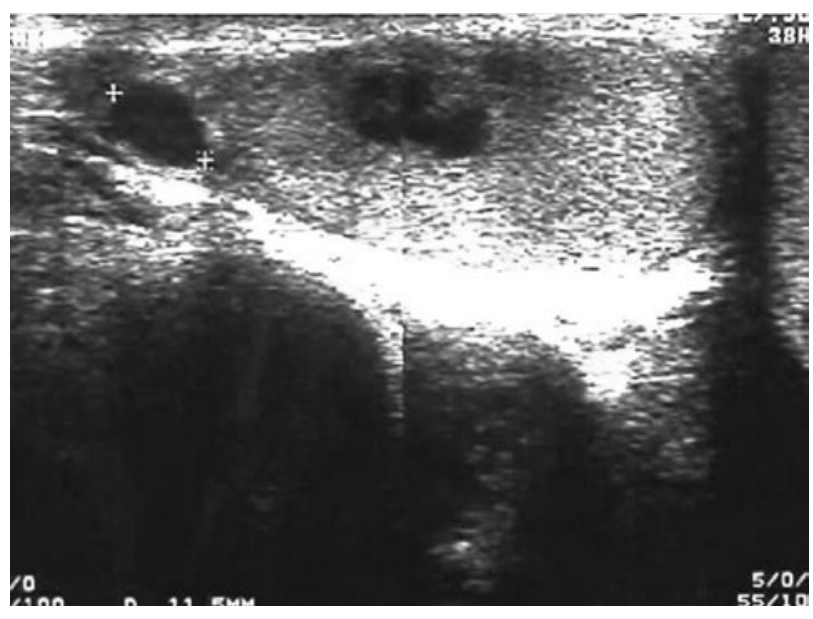

FIGURA 1. Ecografía escrotal con teste ligeramente aumentado de tamaño con área hipoecoica focal de localización periférica. de Bengala y serología positiva para Brucella. El tratamiento que se efectúa es idéntico al caso anterior. La evolución fue satisfactoria.

\section{COMENTARIOS}

La brucelosis, también conocida como fiebre de Malta o fiebre Mediterránea (2), es una enfermedad de baja incidencia y prevalencia en nuestro medio debido fundamentalmente al mejor control en la prevención de enfermedades de transmisión animal. Antaño se asociaba a alta morbilidad en áreas endémicas como el Mediterráneo y Oriente Próximo, como Turquía $(2,4)$, donde puede llegar a una incidencia de 20 casos cada 100.000 habitantes (8). Dentro de la península la incidencia es desigual con una mayor incidencia en la meseta y el sur de la misma, y menor en las regiones del norte. Canarias aparece históricamente libre de brucelosis (8).

La brucelosis fue descrita por primera vez en 1859 por Marston, aunque debe su nombre a Sir David Bruce, quien en 1886 la renombró en lugar de fiebre de Malta (3). Hardy, en 1928, describió su primer caso de epididimo-orquitis brucelar $(5,7,10)$.

Es una enfermedad sistémica producida por el gérmen Brucella, siendo la especie más aislada la Brucella Mellitensis, que es la de mayor contagiosidad y patogenicidad $(8,10)$, seguido en orden de frecuencia por B. Abortus, B. Suis, B. Canis, B. Ovis, B. Neotomae y el recientemente descubierto $B$. Maris (2). Constituye el ganado, así como algunas especies domésticas, su principal reservorio, y es una enfermedad de carácter casi exclusivamente profesional (8), de hecho la mayor prevalencia de esta enfermedad aparece en matarifes, granjeros, pastores de comunidades rurales, veterinarios y gente que consume leche no pasteurizada o queso de éste origen (2). Son cocobacilos, gramnegativos, inmóviles, no esporulados, aerobios, catalasa y oxidasa positivos (8).

La brucelosis se transmite a través del tracto gastrointestinal tras el consumo de carne, leche o productos derivados contaminados, a través del contacto directo con tejidos infectados, bien por inoculación o inhalación, sangre, linfa o piel dañada, a través del tracto respiratorio tras la inhalación en laboratorios microbiológicos, o a través de la conjuntiva $(2,3,6,8)$. No hay evidencia del contagio entre personas (8).

Su presentación clínica compone un amplio espectro de variedades (4). La más habitual es un síndrome febril, inespecífico, ondulante, con sudoración nocturna y pérdida de peso, con afectación de diferentes órganos y sistemas entre los que se encuentra el genitourinario. La afectación local de la enfermedad se describe en el $30 \%$ de los casos (3), con mayor predominancia unilateral (8). Es menos frecuente la forma de debut con afectación genitourinaria que la forma de afectación sis- 
témica. El diagnóstico diferencial incluye otras enfermedades febriles agudas, como la infección por influenza - la tularemia, así como mononucleosis, tuberculosis, enfermedad de Hodgkin's, malaria, infección por HIV, Histoplasmosis o Coccioidomicosis $(2,6,9)$.

Las complicaciones más frecuentes son de localización ósea o articular con una frecuencia de entre el 20 y el $60 \%(2,3)$, tales como espondilitis, artritis supurativa, absceso paraespinal, osteomielitis, tenosinovitis y bursitis (4); hepatoesplenomegalia, linfadenopatías, endocarditis o meningoencefalitis. Las articulaciones con más frecuencia afectadas son la sacroilíaca, rodilla, cadera, muñeca y tobillo (4).

La afectación genitourinaria por la brucelosis, segunda después de la afectación osteoarticular (5), con su amplio abanico de posibilidades: epididimoorquitis, prostatitis, cistitis, nefritis intersticial, pielonefritis, nefropatía $\lg \mathrm{A}$, glomerulonefritis exudativa y absceso renal, es de poco frecuente como forma de presentación de una brucelosis, con una frecuencia estimada de entre el $2-40 \%$ $(2,5)$. La orquiepididimitis de causa brucelar es una forma infrecuente de infección localizada en el testículo y/o epididimo, apareciendo en un $2-20 \%$ de todos los pacientes afectos de brucela $(2,4,7,8,10)$. Su resultado puede ser bien por la eliminación por orina del gérmen o por las metástasis sépticas de origen hemático u óseo (2). El dolor escrotal apareció en el 100\% de los casos $(4,7)$, al tiempo que la fiebre en tan solo algo más de la mitad de los casos (7). La media de edad de los pacientes afectos fue de aproximadamente 37 años y fue significativamente menor que los no afectos de orquiepididimitis (7).

La orquiepididimitis unilateral es la forma más frecuente de presentación (1). Suele ser de comienzo gradual, sin datos inflamatorios muy evidentes, de mayor duración y si sintomatología urinaria asociada (5). La orquitis como entidad sindrómica es a menudo tratada y seguida de forma ambulatoria, excepto las presentaciones con fiebre, afectación del estado general y/o complicación local del cuadro. Es por ello que ante una orquitis de rebelde al tratamiento convencional y de evolución insidiosa, en áreas endémicas y con un probable contacto, bien por su lugar de residencia o por su profesión, debe hacernos sospechar el origen brucelósico (1). El diagnóstico se basa en el aislamiento de Brucella spp. La prueba serológica Rosa de Bengala supone un método diagnóstico rápido y eficaz, y es una prueba de screening muy útil $(1,9)$, con resultado positivo a partir de títulos superiores a $1: 160(2,5,6,10)$. Otras pruebas serológicas son la seroaglutinación de Wright y el test de Coombs anti-Brucella, positivo a partir de títulos superiores a 1:320 $(5,10)$. La negatividad del test de Coombs excluye el diagnóstico (8). El hemograma no suele cursar con leucocitosis, tan solo un aumento de la V.S.G., descenso de la hemoglobina, como resultado de una infección prolongada (10) y alteraciones en la función hepática (5). El estudio de la orina suele ser nor- mal $(2,5,7,10)$, así como la urografía intravenosa (5). La ecografía testicular tiene sus hallazgos típicos como el aumento del tamaño epididimotesticular, descenso de ecogenicidad (6) con hidrocele reaccional y engrosamiento de cubiertas escrotales (5) y al tiempo nos permite descartar la evolución abscesificante o necrotizante $(8,10)$ de la enfermedad cuando observamos en este contexto áreas hipoecoicas bien definidas $(1,2,5)$. En este caso es obligado el diagnóstico diferencial con orquiepidimitis de otro origen o lesiones tumorales. Los hallazgos ecográficos pueden reflejar la severidad de la afección (6). La infección limitada al testículo es rara, suele envolver al epidídimo, y en estos casos, puede afectar bien a la totalidad o bien una parte, siendo la cabeza y cola del epidídimo los más frecuentes $(6,10)$. En casos de afectación epididimaria pura, el testículo tiene una normal ecogenicidad, ecoestructura y flujo sanguíneo (6). El hidrocele reaccional es anecoico y puede tener similar aspecto al hematocele, en relación con traumatismos o tumores, o piocele, resultado de epididimoorquitis mal tratadas o ruptura de un absceso intratesticular $(6,10)$. Además, se ha descrito el uso de la ultrasonografía con escala de grises y doppler-color, así como los estudios con radionúclidos sobre el teste para ayudar a diferenciar esta rara infección testicular de otras entidades que cursan con el síndrome de "enlarged scrotum", tanto benignas como la torsión, el traumatismo o hematocele, como malignas $(2,3)$. Si tras la torsión, ocurre una detorsión espontánea, no es posible diferenciar el cuadro de una epididimoorquitis mediante Ecografía (6). En determinados pacientes el diagnóstico de brucelosis se ha efectuado tras la orquiectomía por sospecha de enfermedad maligna (3). Es característico que el engrosamiento de la piel no aparece en los procesos malignos (6). El uso de estas tecnologías contribuye a disminuir el número de orquiectomías innecesarias (2). El diagnóstico de orquiepidididmitis brucelósica frente a otras causas más frecuente de orquiepididimitis debe basarse en la duración de la sintomatología, la naturaleza subaguda, las características de la fiebre y la ausencia de leucocitosis (3). Los hemocultivos completan el estudio diagnóstico de la patología. Los hemocultivos pueden ser positivos en el $50 \%$ de los pacientes con brucelosis aguda a las dos o tres semanas (2), confirman el diagnóstico $(8,10)$. Al tiempo que los hemocultivos, el cultivo del aspirado espermático puede revelar la presencia del microorganismo (10). La brucelosis puede disminuir el recuento espermático y ser causante de una posible infertilidad (7). Recientemente se han descrito técnicas con PCR para el diagnóstico de las complicaciones locales de la enfermedad. El examen histopatológico revela infección granulomatosa no caseificante con infiltrado de predominio linfohistiocitario $(7,10)$.

El tratamiento médico más aceptado sigue siendo la combinación de tetraciclinas (Doxiclina $100 \mathrm{mg}$. cada 12 horas durante 45 días) con Rifampicina (600-900 $\mathrm{mg} . /$ día durante al menos 6 semanas) $\circ$ Estreptomicina (1 gr. i.m. al día durante 2-3 semanas), con una evolución aceptable en un importante número de casos 
$(1,4)$. Se acepta el inicio del tratamiento en pacientes con alta sospecha clínica de la enfermedad a la espera de la confirmación definitiva $(2,8)$. No se recomienda regímenes con una sola droga dada la alta tasa de reaparición de un cuadro de brucelosis sistémica, tasada en más del $50 \%$ (2). En caso de recidiva del cuadro se requiere nuevos ciclos antibióticos y de mayor duración. En caso de orquiepididimitis necrotizante o resistente al tratamiento, se recomienda la orquiectomía $(4,5,7,9)$, que según series oscila en torno al $8 \%(10)$.

La brucelosis en la infancia ha sido considerada como una entidad muy rara, pero recientes estudios demuestra que puede llegar a ser normal en áreas donde el germen es endémico, y podría estar implicada en la infertilidad primaria en estas zonas (10).

\section{CONCLUSIONES}

La brucellosis es todavía un problema de salud pública en los países endémicos, especialmente el Sur de Europa y determinadas comunidades en desarrollo, aunque poco frecuente. La orquiepididimitis es una entidad relativamente frecuente y un manejo inapropiado con la administración precoz de terapia antibiótica combinada puede derivar en serias complicaciones como absceso testicular, infertilidad masculina o atrofia de testes. En todo escroto agudo con antecedentes sospechosos de contagio y, en especial en áreas endémicas, debe iniciarse el tratamiento apropiado sin esperar a la confirmación serológica. El test aglutinación podría ser de ayuda, junto a la Ecografía Doppler-Color en la investigación de la orquiepididimitis en áreas endémicas o ante paciente que asocien el escroto agudo a dolor articular y fiebre con escalofríos.

\section{BIBLIOGRAFÍA y LECTURAS RECOMENDADAS (*lectura de interés $y^{* *}$ lectura fundamental)}

1. VALDELVIRA NADAL, P.; NICOLÁS TORRALBA, J.A.; BAÑ́́N PÉREZ, V.J. y cols.: "Orquiepididimitis brucelosa". Actas Urol. Esp. 25:140, 2002.

*2. PAPATSORIS, A.G.; MPADRA,F.A.; KARAMOUZIS, M.V. y cols.: "Endemic brucellar epididymo-orchitis: a 10-year experience". Int. J. Infect. Dis.; 6:309, 2002.

*3. AKRITIDIS, N.; MASTORA, M. y PAPPAS, G.: "Genitourinary complications of brucelosis"; Infections in Medicine; 19: 384, 2002.

4. CESUR, S.; ÇIFTÇI, A.; SÖZEN, T.H. y cols. : “A case of epididymo-orchitis and paravertebral abscess due to Brucellosis". J. of Infec.: 251, 2002.

5. PÉREZ FENTES, D.; BLANCO PARRA, M.; ALENDE SIXTO, M. y cols.: "Orquiepididimitis: A propósito de un caso; Arch. Esp. Urol., 58: 674, 2005.

*6. OZTURK, A.; OZTURK, E.; ZEYREK, F. y cols. : "Comparison of brucella and non-specific epididymorchitis: gray scale and color Doppler ultrasonographic features"., 56: 256, 2005.

*7. AKINCI, E.; BODUR, H.; ÇEVIK, M.A. y cols. : “A complication of brucelosis: Epididymoorchitis". Int. J. Inf. Disease, 10: 171, 2006.

8. ALAPONT ALACREU, J.M.; GÓMEZ DELGADO, L. y DELGADO, F.: "Orquiepididimitis por brucella; Actas Urol. Esp., 28 : 774, 2004.

**9. GUINDA SEVILLANO, C.; AREVALO VELASCO, J.M. y PÉREZ ARBEJ, J.A. : "Orquitis brucelar. Aportación de una serie de 16 casos". Actas Urol. Esp., 19: 455, 1995.

**10. NAVARRO MARTÍNEZ, A.; SOLERA, J. y BEATO, J.L.: "Epididymoorchitis due to brucella mellitensis: a retrospectice study of 59 patients". Clinical Infectious Disease, 33: 2017, 2001.

Casos Clínicos

Arch. Esp. Urol., 60, 7 (795-799), 2007

\section{BILHARZIASIS: PRESENTACIÓN DE UN CASO CLÍNICO.}

\begin{abstract}
Luis Labairu Huerta, José Ángel Cuesta Alcalá, Saturnino Napal Lecumberri, Marisa Gómez Dorronsoro' y Juan Ignacio Pascual Piédrola.
\end{abstract}

Servicio de Urología y Servicio de Anatomía Patológica', Hospital de Navarra. Pamplona. España.

\begin{abstract}
Resumen.- OBJETIVO: Presentar un caso clínico atendido en nuestro centro y comentar brevemente la literatura en el contexto actual del incremento de las enfermedades parasitarias en Europa, importadas del tercer mundo con la inmigración y el turismo.
\end{abstract}

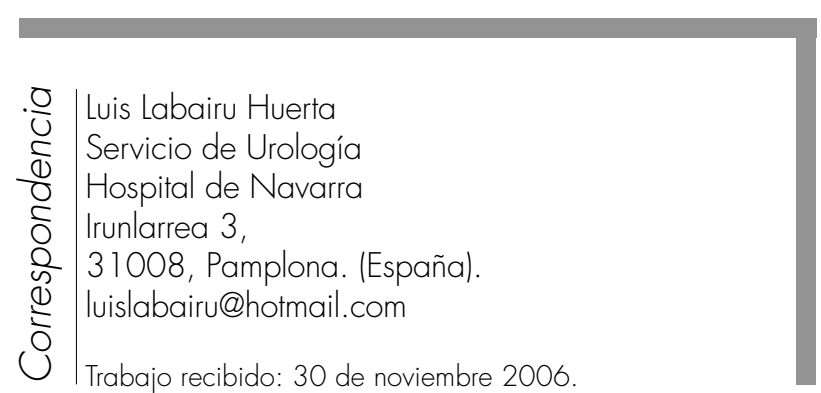

